Study of the Relation between Hypoxia and Muscle Atrophy

Caron Marc-André, Marie-Ève Paré, François Maltais, Richard Debigaré, Unité de recherche en pneumologie, Hôpital Laval, Québec, Canada

Background : Skeletal muscle atrophy is an important feature of chronic obstructive pulmonary disease (COPD) and it is recognized to have considerable clinical impacts. Unfortunately, factors contributing to muscle wasting in COPD are poorly understood. Hypoxemia is typical in COPD and several evidences link hypoxic conditions and protein breakdown. We propose that hypoxia participate to muscle atrophy by increasing Ubiquitin-Proteasome (UP) system activity and by decreasing the activity of IGF/PI3K/Akt synthesis pathway.

Methods: To test this hypothesis, L6 muscle myotubes were either exposed to hypoxia $\left(1 \% \mathrm{O}_{2}\right)$ or normoxia $\left(21 \% \mathrm{O}_{2}\right)$.

Results: After 24 hours of hypoxic exposure, we found a significant rise in the chymotrypsin and caspase-like 20S proteasome activities. Proteolysis was confirmed by an accumulation of a $14 \mathrm{kDa}$ actin fragment during hypoxia. An elevation of Atrogin-1 mRNA expression was also observed in similar conditions. A decline in Akt phosphorylation was noticed in hypoxia. These changes were attenuated by insulin treatment.

Conclusion: Proteolysis is accentuated in myotubes exposed to hypoxia and the UP system appears to be involved. In addition, protein synthesis seems to be affected as a lower Akt activity was observed. However, the IGF/PI3K/Akt pathway can still be stimulated by a suitable signal suggesting that therapies targeting this pathway are conceivable.

\section{Predictive Value of the Transtheoretical Model to Smoking Cessation in Hospitalized Patients with Cardiovascular Disease}

\author{
Chouinard, Maud-Christine ; Robichaud-Ekstrand, \\ Sylvie, Université du Québec à Chicoutimi, Chi- \\ coutimi, Québec, Canada. Université de Moncton, \\ Moncton, New Brunswick, Canada
}

Background: Several authors have questioned the Transtheoretical Model (TTM). Determining the predictive value of each cognitive-behavioural element within this model could explain the successes reported in smoking cessation programs. The purpose of this study was to predict point-prevalent smoking abstinence and progression through stages of change at 2 and 6 months, using the constructs of the TTM, when applied to a pooled sample of individuals who were hospitalized for a cardiovascular event.

Methods: In a predictive correlation design, recently hospitalized patients $(\mathrm{N}=168)$ with cardiovascular disease (CVD) were pooled from a randomized controlled trial. Independent variables of the predictive TTM comprise stages and processes of change, pros and cons to quit smoking (decisional balance), selfefficacy, and social support. These were evaluated at baseline, 2 and 6 months.

Results: Compared with smokers, individuals who abstained from smoking at 2 and 6 months were more confident at baseline to remain non-smokers, perceived less pros and cons to continue smoking, utilized less consciousness raising and self-reevaluation experiential processes of change, and received more positive reinforcement from their social network with regard to their smoke-free behaviour. Self-efficacy at baseline was the only element which predicted that patients would progress through the stages of change between hospital discharge and 6 months.

Conclusions: Self-efficacy was the only element which predicted smoking abstinence and progression through stages of change. Observations about the other elements are congruent with the TTM. This study provides important information regarding the application of the TTM to smoking cessation in CVD patients. 